Optimizing piezoelectric transformer for maximum power transfer

This article has been downloaded from IOPscience. Please scroll down to see the full text article.

2003 Smart Mater. Struct. 12373

(http://iopscience.iop.org/0964-1726/12/3/308)

The Table of Contents and more related content is available

Download details:

IP Address: 140.112.113.225

The article was downloaded on 25/12/2008 at 02:18

Please note that terms and conditions apply. 


\title{
Optimizing piezoelectric transformer for maximum power transfer
}

\author{
Yu-Hsiang Hsu ${ }^{1}$, Chih-Kung Lee ${ }^{1,3}$ and Wen-Hsin Hsiao ${ }^{2}$ \\ ${ }^{1}$ Institute of Applied Mechanics, National Taiwan University, No 1, Section 4, Roosevelt Road, \\ Taipei, Taiwan 106, Republic of China \\ ${ }^{2}$ AdvanceWave Technology, Incorporated, Taipei, Taiwan 106, Republic of China \\ E-mail: cklee@mems.iam.ntu.edu.tw
}

Received 5 September 2002, in final form 31 March 2003

Published 28 April 2003

Online at stacks.iop.org/SMS/12/373

\begin{abstract}
The piezoelectric transformer should be considered as not only a mechanical system but also an electrical fully coupled system, which uses finite structure resonance to perform voltage conversion. The traditional piezoelectric transformer uses an equivalent circuit concept to simulate its mechanical motion. Since the field equation of a piezoelectric transformer is a partial differential equation, an equivalent circuit developed based on an ordinary differential equation cannot express its performance thoroughly. Since the motion of the piezoelectric transformer is determined by both the mechanical and the electrical boundary conditions, the transfer function and electrical impedance of a piezoelectric transformer will vary with different electrical and mechanical conditions. However, it has been discovered that once an equivalent circuit is used to simplify the mechanical motion of the piezoelectric transformer, the coupling effects between the mechanical and the electrical properties will be averaged out and all spatial information will be lost. A fully coupled field equation is derived in this paper to examine the motion of the piezoelectric transformer by taking into account both the mechanical and electrical boundary conditions. It can be shown that by using this newly developed field equation, a new concept using a weighting function in the spatial domain can be adopted to optimize the electro-mechanical coupling effects. In this paper, both impedance and power transfer optimization of the source as well as the sink of the piezoelectric transformer, and the corresponding interface circuits, are verified theoretically and experimentally.
\end{abstract}

\section{Introduction}

Piezoelectric transformers were first invented by Rosen in $1956[1,2]$. At that time, the fundamental concept was based on using a finite structure resonance mode in bulk motion to transfer a low AC input voltage to a high AC output voltage. Since the quality factor of a piezoelectric ceramic is easily in the range of one to two thousands, the transfer loss of a piezoelectric transformer is naturally much lower than that of an electromagnetic transformer or a resonance tank by a RLC circuit [3]. More specifically, the quality factor for a RLC-circuit-based electrical resonance tank is

3 Author to whom any correspondence should be addressed. merely around 10-20, which is two orders of a magnitude lower than that for a mechanical-based resonator such as a piezoelectric transformer. In addition to possessing high efficiency, piezoelectric transformers are considered one of the most important power transfer devices for the next generation due to the low profile, no EMI noise, and no potential short-circuit fire hazard such as found in electromagnetic transformers. Many research organizations including both academic organizations and high-tech companies have injected many resources into the study of piezoelectric transformers in the hope of capitalizing on the advantages of these transformers. The most representative inventions in the field that have come out from such R\&D efforts have been (1) third- 
order longitudinal mode piezoelectric transformers by Kawai et al [4], (2) alternately poled piezoelectric transformers by Kanayama et al [5], and (3) central drive-type half-lambda mode piezoelectric transformer by Tooru et al [6].

Despite possessing excellent characteristics and showing promising R\&D results, piezoelectric transformers still only account for a small portion of the power supply market even after 50 years of development. With the ever-expanding of the cold cathode fluorescent lamp (CCFL) backlight market for liquid crystal displays and the ever more important heat management of these displays, piezoelectric transformers are finally making some headway into this application even though its market percentage still remains insignificant. It will be shown in this paper that a fundamental paradigm change in the modeling of the mechanical motion of the piezoelectric transformer must be introduced in order to further advance the art of piezoelectric transformers.

It should be noted that all of the above-mentioned piezoelectric transformers were basically a rearrangement of the original Rosen-type piezoelectric transformer, which really did not break free of the limitations embedded within the mechanical design concept of the original Rosen-type transformers. At the same time, equivalent circuits [1, 7] were the primary electric design parameters for piezoelectric transformers. It will be clearly demonstrated herein that adopting the equivalent circuit concept, which was developed by using ordinary differential equations (ODEs) to simulate the motion of the piezoelectric transformer, does not optimize the coupling effects between the mechanical motion and the corresponding electrical impedance match by using the interface circuit. More specifically, it will be shown that optimization can be achieved by using the partial differential equations (PDEs), i.e. the wave equations, to examine the motion of the piezoelectric transformer structures.

A general field equation suitable to model the wave propagation nature of both single- and multi-layer piezoelectric transformers was first derived to take full consideration of the mechanical and electrical boundary conditions. With the coupling phenomenon between the mechanical resonance and the electrical power transfer fully analyzed, requirements for the operation topology of piezoelectric transformers can be easily identified. On the other hand, the concept of modal sensors and actuators [8] that are typically adopted in the field of flexible structure control will also be shown to be applicable here. Theoretical derivations and experimental results, both of which lead to the conclusion that optimal power transfers can be achieved for piezoelectric transformers based on the newly derived field equations, will be presented in detail.

\section{Theory of piezoelectric plates}

Piezoelectricity, which was discovered by Pierre Curie and Jacques Curie [9], relates to the conversion between electrical and mechanical energies. Its constitutive equations can be expressed as [10]

$$
\begin{gathered}
T_{i j}=c_{i j k l}^{E} S_{k l}-e_{k i j} E_{k} \\
D_{j}=e_{j k l} S_{k l}+\varepsilon_{j k}^{s} E_{k}
\end{gathered}
$$

where $i, j, k, l=1-3$. Equation (1a) shows that both a mechanical strain $S_{k l}$ and an electrical field $E_{k}$ can induce a mechanical stress $T_{i j}$ through elastic constant $c_{i j k l}^{E}$ and piezoelectric stress constant $e_{k i j}$, respectively. This equation represents an actuation effect of piezoelectricity, which means that a mechanical deformation can be generated by applying an electrical field. On the other hand, equation $(1 b)$ shows that both a mechanical strain $S_{k l}$ and an electrical field $E_{k}$ can induce an electrical displacement $D_{j}$ through piezoelectric stress constant $e_{j k l}$ and permittivity constant $\varepsilon_{j k}^{s}$, respectively. This equation essentially shows that piezoelectricity can build up electrical charges on the surfaces when mechanical strains are applied. Note that the superscripts ' $S$ ' and ' $E$ ' relating to the material constants are used to indicate that these material constants were obtained by keeping the $S$ or $E$ fields constant.

The governing equations of the piezoelectric material thus become [11]

$$
\begin{gathered}
T_{i j, j}=\rho \ddot{u}_{i}, \\
D_{j, j}=0,
\end{gathered}
$$

where $u$ represents the displacement field. The typical piezoelectric material used to implement piezoelectric transformers belongs to the class of material based on lead, zirconate and titanate (PZT), which possess a class $6 \mathrm{~mm}$ symmetry crystal system. Substituting the constitutive equation of PZT into equations $(2 a)$ and $(2 b)$ in terms of IEEE compact matrix notation leads to [11]

$$
\begin{aligned}
c_{11}^{E} S_{1,1} & +c_{12}^{E} S_{2,1}+c_{13}^{E} S_{3,1}+c_{66}^{E} S_{6,2}+c_{44}^{E} S_{5,3} \\
& +e_{31}\left(\varphi_{, 3}\right)_{, 1}+e_{15}\left(\varphi_{, 1}\right)_{, 3}=\rho \ddot{u}_{1} \\
c_{11}^{E} S_{2,2} & +c_{12}^{E} S_{1,2}+c_{13}^{E} S_{3,2}+c_{66}^{E} S_{6,1}+c_{44}^{E} S_{4,3} \\
& +e_{31}\left(\varphi_{, 3}\right)_{, 2}+e_{15}\left(\varphi_{, 2}\right)_{, 3}=\rho \ddot{u}_{2} \\
c_{33}^{E} S_{3,3} & +c_{13}^{E}\left(S_{1,3}+S_{2,3}\right)+c_{44}^{E}\left(S_{5,1}+S_{4,2}\right)+e_{15}\left(\varphi_{, 1}\right)_{, 1} \\
& +e_{15}\left(\varphi_{, 2}\right)_{, 2}+e_{33}\left(\varphi_{, 3}\right)_{, 3}=\rho \ddot{u}_{3}
\end{aligned}
$$

and

$$
\begin{aligned}
& e_{33} S_{3,3}+e_{31}\left(S_{1,3}+S_{2,3}\right)+e_{15}\left(S_{5,1}+S_{4,2}\right) \\
& \quad-\left(\varepsilon_{11}^{s}\left(\varphi_{, 1}\right)_{, 1}+\varepsilon_{11}^{s}\left(\varphi_{, 2}\right)_{, 2}+\varepsilon_{33}^{s}\left(\varphi_{, 3}\right)_{, 3}\right)=0,
\end{aligned}
$$

where $\varphi$ is the electrical potential. It should be mentioned that the subscripts $i=1-3$ of $\varphi$ in the brackets in equation (3) are the subscript $k$ of the electrical field $E$ in equation (1), which shows the direction of the electrical fields. The subscripts outside of the brackets of $\varphi$ in equation (3) are the electrical contributions to the displacement field from the governing equations (2). Note that only the equation of motion for the displacement field $u_{3}$ is coupled to the charge equation derived from the electrostatics equation, i.e. equation $(3 b)$. That is, the piezoelectric effect will stiffen only the elastic constants that contribute to the $u_{3}$ displacement field [11]. On the other hand, the piezoelectric effect serves as an inhomogeneous term in the displacement field of $u_{1}$ and $u_{2}$ without influencing the stiffness of the elastic constant. The piezoelectric transformer invented by Rosen $[1,2]$ was based on using the governing equation of the $u_{1}$ displacement field to serve as the actuator, and the governing equation of the $u_{3}$ displacement field as the sensor portion. Applying the concept of modal sensors and actuators, the governing equations of a piezoelectric plate similar to the one shown in figure 1(a) can be seen. 


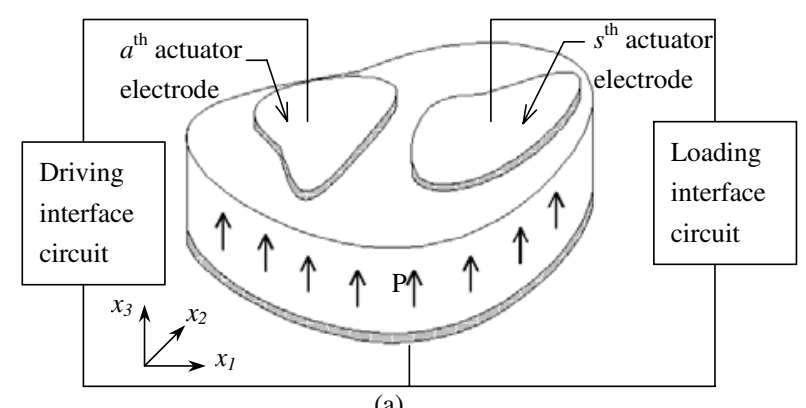

(a)

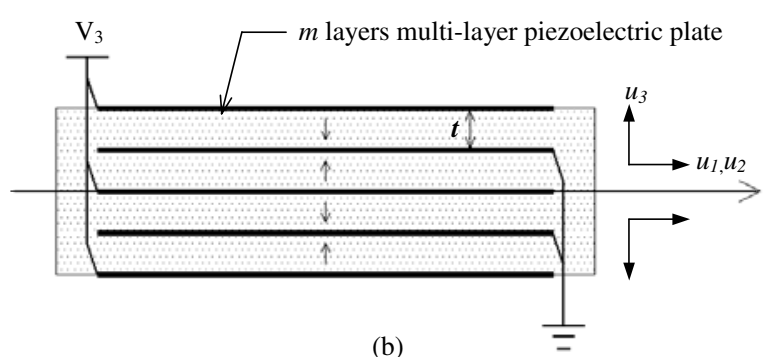

Figure 1. (a) Schematic of a piezoelectric plate, (b) schematic of a multi-layer piezoelectric plate with $m$ layers.

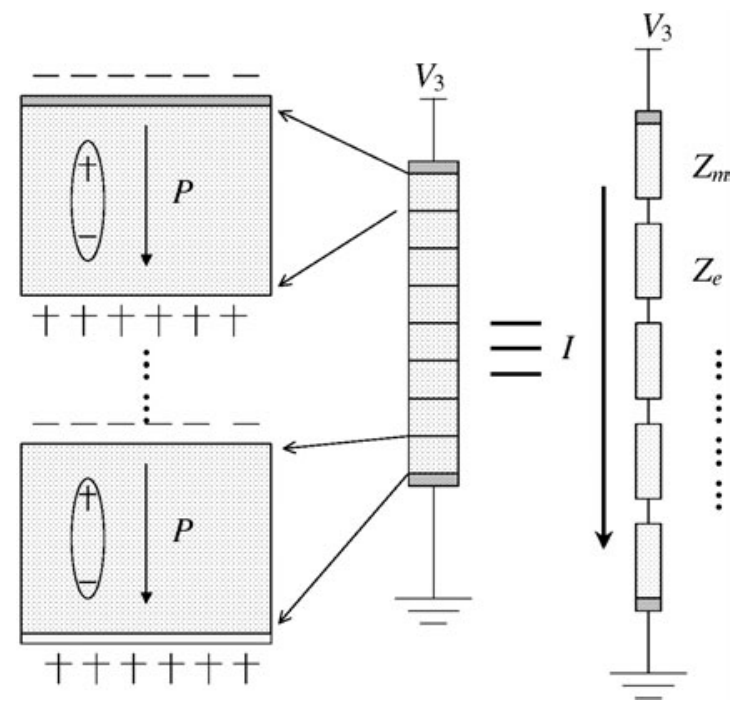

Figure 2. Schematic of the physical meaning of the averaged constitutive equation for a single-layer piezoelectric plate.

\subsection{Two-dimensional constitutive equation of a piezoelectric plate}

Three basic assumptions were made for the piezoelectric plate:

(1) the thickness of the piezoelectric plate is much thinner than its width and length,

(2) the thickness of the electrodes are much thinner than the plate thickness, and

(3) its displacement fields are symmetric with respect to its neutral plane (figure 1(b)).

Placing the physical coordinates of the piezoelectric plate on the neutral plane, and averaging its physical parameters with respect to the $x_{3}$ axis, we obtain

$$
\tilde{P}=\int_{-h / 2}^{h / 2} P(x, y, z) \mathrm{d} z,
$$

where $\tilde{P}$ represents the averaged physical parameter $P$ and $h$ is the thickness of the piezoelectric plate. The averaged two-dimensional strains $\tilde{S}_{4}$ and $\tilde{S}_{5}$ were zero, and $\tilde{S}_{3}$ was approximated to be zero as the thickness is thinner with respect to all lateral scales. Based on these assumptions and approximations, the reduced two-dimensional piezoelectric constitutive equations become

$$
\begin{gathered}
\tilde{T}_{1}=c_{11}^{E} \tilde{u}_{1,1}+c_{12}^{E} \tilde{u}_{2,2}-m e_{31}\left(V_{3}\right) \\
\tilde{T}_{2}=c_{12}^{E} \tilde{u}_{1,1}+c_{11}^{E} \tilde{u}_{2,2}-m e_{31}\left(V_{3}\right) \\
\tilde{T}_{6}=c_{66}^{E}\left(\tilde{u}_{1,2}+\tilde{u}_{2,1}\right),
\end{gathered}
$$

and

$$
m t \tilde{D}_{3}=e_{31} \tilde{u}_{1,1}+e_{31} \tilde{u}_{2,2}+m \varepsilon_{33}^{s}\left(V_{3}\right),
$$

where $m$ is a piezoelectric plate with a total of $m$ layers, and $t$ is the thickness of one of the piezoelectric layers of interest. If $m=1$, it denotes a single-layer piezoelectric plate with a thickness $t$. The physical meaning of the constitutive equation (equation $(5 b)$ ) can be better understood by substituting the relationship of the constants as

$$
\begin{gathered}
m\left(V_{3}\right)=-\frac{e_{31}}{\varepsilon_{33}^{s}} \tilde{u}_{1,1}-\frac{e_{31}}{\varepsilon_{33}^{s}} \tilde{u}_{2,2}+\frac{1}{\varepsilon_{33}^{s}} m t \tilde{D} \\
=-h_{31} \tilde{u}_{1,1}-h_{31} \tilde{u}_{1,1}+\beta_{33}^{s} m t \tilde{D}_{3} .
\end{gathered}
$$

Considering figure 2 , which is a single-layer piezoelectric plate, the average of the electrical field $E_{3}$ along the $x_{3}$ axis equals the external voltage $V_{3}$ between the electrodes, and the current is induced by the distributed charges through a series of electrical and mechanical equivalent impedances $Z_{e}$ and $Z_{m}$, respectively. If this is a multi-layer piezoelectric plate with $m$ layers, each layer will have voltage potential equal to the external voltage $V_{3}$, and the summation of charges of all layers will equal the integration of all external current with respect to time, which is nothing but a representation of the Kirchhoff voltage and current theorem [12].

\subsection{Governing equation of piezoelectric plate}

Averaging the governing equation of a piezoelectric plate in (2) with equation (4), and then substituting equation (5) to the resultants leads to the governing equations of a twodimensional piezoelectric plate

$$
\begin{aligned}
& c_{11}^{E} \tilde{u}_{1,11}+\frac{c_{11}^{E}+c_{12}^{E}}{2} \tilde{u}_{2,21}+\frac{c_{11}^{E}-c_{12}^{E}}{2} \tilde{u}_{2,11}-e_{31}\left(V_{3}\right)_{, 1}=\rho \tilde{\ddot{u}}_{1} \\
& c_{11}^{E} \tilde{u}_{2,22}+\frac{c_{11}^{E}+c_{12}^{E}}{2} \tilde{u}_{1,12}+\frac{c_{11}^{E}-c_{12}^{E}}{2} \tilde{u}_{1,22}-e_{31}\left(V_{3}\right)_{, 2}=\rho \tilde{\ddot{u}}_{2},
\end{aligned}
$$

where $c_{66}^{E}=\left(c_{11}^{E}-c_{12}^{E}\right) / 2$ has been applied. Note that equation (7) shows that the class $6 \mathrm{~mm}$ symmetric piezoelectric material is transversely isotropic in the $x_{1}-x_{2}$ plane [13]. The one-dimensional piezoelectric plate shown in figure 3 has multiple inputs and multiple output electrodes in the $x_{1}-x_{2}$ plane and free-free boundary conditions. Assuming that the 
length $l$ of the piezoelectric plate is an order of magnitude larger than width $w$, and the displacement fields depend on the $x_{1}$ coordinate only, i.e. long-wavelength assumption, the governing equation and the constitutive equations can be further reduced to

$$
c_{11}^{E} \tilde{u}_{1,11}-e_{31}\left(\sum_{a=1}^{A} m_{a} V_{3 a}^{\text {in }}+\sum_{s=1}^{S} m_{s} V_{3 s}^{\text {out }}\right)_{, 1}=\rho \tilde{\ddot{u}}_{1},
$$

and

$$
\begin{gathered}
\tilde{T}_{1}=c_{11}^{E} \tilde{u}_{1,1}-m e_{31}\left(V_{3}\right) \\
m t \tilde{D}_{3}=e_{31} \tilde{u}_{1,1}+m \varepsilon_{33}^{s}\left(V_{3}\right),
\end{gathered}
$$

where subscripts $a$ and $s$ represent the $a$ th actuator and the $s$ th sensor, respectively. Equation $(8 a)$ is the generalized governing equation of the one-dimensional piezoelectric plate with multi-input and multi-output electrodes. Equations (8b) are the constitutive equations for actuator and sensor. From equation (8), it is clear that the action of the piezoelectricity, whether for the sensor or the actuator, can be viewed as a form of the external forces applied onto the one-dimensional plate. Considering the eigenfunction expansion of a one-dimensional governing equation [14]

$$
\frac{\mathrm{d}^{2} \phi_{i}}{\mathrm{~d} x^{2}}+k_{i}^{2} \phi_{i}=0
$$

where

$$
\frac{\mathrm{d} \phi_{i}(0)}{\mathrm{d} x}=\frac{\mathrm{d} \phi_{i}(l)}{\mathrm{d} x}=0,
$$

are the boundary conditions of the eigenfunction, the $i$ th eigenfunction of this free-free one-dimensional piezoelectric plate is thus

$$
\phi_{i}(x)=\sqrt{\frac{2}{l}} \cos \left(k_{i} x\right), \quad i=1 \sim \infty
$$

where $k_{i}=i \pi / l$ is the $i$ th eigenvalue of the $i$ th eigenfunction and represents the wavenumber of the system. Since this is a typical Sturm-Liouville problem, these eigenfunctions form complete sets of the structure system, and possess orthogonal property with respect to each other. Note that a normalized constant $(2 / l)^{1 / 2}$ was added as well. The displacement field of the one-dimensional piezoelectric plate can be shown as the superposition of the eigenfunctions, i.e. $\tilde{u}_{1}(x, t)=\sum_{i=1}^{\infty} A_{i}(t) \phi_{i}(x)$. Taking a volume integration of equation (8a) with respect to the $j$ th eigenfunction, the governing equation, which is a PDE, can be further reduced to an infinite number of ODEs. The $i$ th ODE becomes

$$
\frac{\mathrm{d}^{2} A_{i}(t)}{\mathrm{d} t^{2}}+\frac{c_{11}^{E} k_{i}^{2}}{\rho} A_{i}(t)=r_{i}(t)
$$

where

$$
\begin{aligned}
r_{i}(t) & =-\frac{e_{31}}{\rho} \int_{0}^{l} \frac{\mathrm{d}}{\mathrm{d} x}\left(\sum_{a=1}^{A} m_{a} V_{3 a}^{i n}+\sum_{s=1}^{S} m_{s} V_{3 s}^{\text {out }}\right) \phi_{i}(x) \mathrm{d} x \\
& +\frac{c_{11}^{E}}{\rho}\left[\frac{\partial \tilde{u}(l, t)}{\partial x} \phi_{i}(l)-\frac{\partial \tilde{u}(0, t)}{\partial x} \phi_{i}(0)\right]
\end{aligned}
$$

is the inhomogeneous term of the $i$ th-mode ODE. It is also clear from these equations that the piezoelectric effects of the actuators and sensors can be viewed as distributed forces

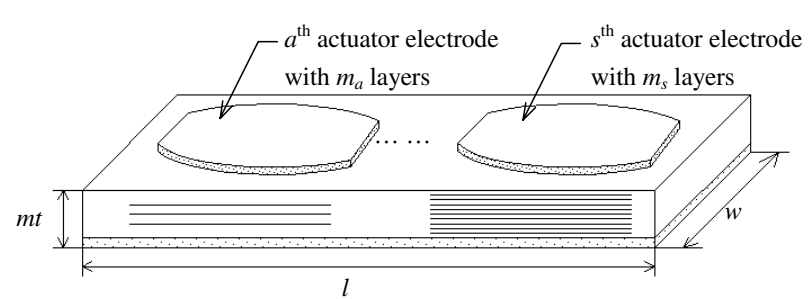

Figure 3. Schematic of a one-dimensional piezoelectric plate with multi-input and -output electrodes, where $a$ and $s$ represent the $a$ th actuator and $s$ th actuator, respectively.

on the piezoelectric plate. In other words, since the actions are performed in the spatial domain, the concept of applying a spatially distributed weighting function to optimize the performance of a piezoelectric transformer is possible. In the next section, it is shown that the concept of modal sensors and actuators [8] can be implemented in piezoelectric transformers. The generalized solution of a one-dimensional piezoelectric plate with multi-input and multi-output electrodes in terms of a displacement field then becomes

$$
\tilde{u}(x, t)=\sum_{i=1}^{\infty} A_{i}(t) \phi_{i}(x)=\sum_{i=1}^{\infty} \frac{r_{i}}{\left(\omega_{i}^{2}-\omega^{2}\right)} \phi_{i}(x),
$$

where $\omega_{i}=\left(C_{11}^{E} k_{i}^{2} / \rho\right)^{1 / 2}$ is the $i$ th resonance frequency of the piezoelectric plate. Since the boundary conditions of this one-dimensional piezoelectric plate are traction free, the second inhomogeneous term in equation (13) equals zero [14]. Equation (14) is the generalized displacement field of the piezoelectric transformer, which will be used to study a singleinput-single-output (SISO) piezoelectric transformer in the next section.

\subsection{SISO piezoelectric transformers}

Considering a SISO piezoelectric transformer with the generalized displacement field from equation (14) with $A$ and $S$ set equal to 1 in equation (13), we obtain

$$
\begin{gathered}
\tilde{u}(x, t)=\sum_{i=1}^{\infty}-\frac{e_{31}}{\rho} \int_{0}^{l} \frac{\mathrm{d}}{\mathrm{d} x}\left(m_{a} V_{3 a}^{i n} A_{a}(x)\right. \\
\left.+m_{s} V_{3 s}^{\text {out }} S_{s}(x)\right) \phi_{i}(x) \mathrm{d} x \frac{\phi_{i}(x)}{\left(\omega_{i}^{2}-\omega^{2}\right)}
\end{gathered}
$$

where the subscripts $a$ and $s$ represent the actuator and sensor of the piezoelectric transformer respectively, and where $A_{a}(x)$ and $S_{S}(x)$ are the effective surface electrodes of the actuator and sensor, i.e. the part of the surface on the piezoelectric layer that has electrodes on both the top and bottom surfaces. It will be shown that effective surface electrodes offer very important design parameters for the design of piezoelectric transformers. Taking surface integration with respect to the actuator and the sensor electrodes from the one-dimensional constitutive equation in equation $(8 b)$ leads us to

$$
\begin{gathered}
\int_{0}^{l}\left(m_{a} t_{a} \tilde{D}_{3 a}^{i n}\right) A_{a}(x) w \mathrm{~d} x=\int_{0}^{l}\left(e_{31} \tilde{u}_{1,1}\right) A_{a}(x) w \mathrm{~d} x \\
+\int_{0}^{l}\left(\varepsilon_{33}^{s} m_{a} V_{3 a}^{i n}\right) A_{a}(x) w \mathrm{~d} x
\end{gathered}
$$




$$
\begin{aligned}
& \int_{0}^{l}\left(m_{s} t_{s} \tilde{D}_{3 s}^{\text {out }}\right) w S_{s}(x) \mathrm{d} x=\int_{0}^{l}\left(e_{31} \tilde{u}_{1,1}\right) w S_{s}(x) \mathrm{d} x \\
& \quad+\int_{0}^{l}\left(\varepsilon_{33}^{s} m_{s} V_{3 s}^{\text {out }}\right) w S_{s}(x) \mathrm{d} x .
\end{aligned}
$$

Since the current flowing in a closed circuit formed by the interface circuit and the piezoelectric plate must equal zero [12], the electrical displacement measured from the interface circuit through the electrodes possesses opposite signs with respect to the one in the solid, which can be derived from the constitutive equations, i.e. the material property of the piezoelectric solid. From Kirchhoff's current theorem, the current flow into or out of a multi-layer piezoelectric plate equals the summation of the currents generated within each layer. That is, the current that is injected into a multi-layer actuator will be spread to each layer to drive the piezoelectric transformer. As for the sensor, the current flowing into the loading impedance of a multi-layer sensor will equal the charges generated by all of the layers. Physically, the configuration of a multi-layered piezoelectric laminate corresponds to a parallel connection of equivalent resistances. That is, each of the layers possesses identical voltage and shares the same external current. From a generalized Ohm's law, $V_{3 a}^{\text {in }}$ and $V_{3 s}^{\text {out }}$ can be represented by $I_{3 a}^{\text {in }} Z_{e}$ and $I_{3 a}^{\text {out }} Z_{L}$ respectively, where $Z_{e}$ and $Z_{L}$ are the equivalent input impedance and external loading. The input impedance of the SISO piezoelectric transformer can be derived from equations $(16 a)$ and $(16 b)$ as

$$
\begin{aligned}
Z_{e}= & \sum_{i=1}^{\infty} \frac{-1}{j \omega c_{a}} \\
& \times \frac{\left(\omega_{i}^{2}+2 \zeta(j \omega) \omega_{i}-\omega^{2}\right)-m_{s} \frac{w}{t_{s}} \frac{j \omega Z_{L} C_{s}}{1+j \omega Z_{L} C_{s}} \frac{e_{31}^{2}}{\rho C_{s}} S_{1} S_{2}}{\left(\omega_{i}^{\prime 2}+2 \zeta(j \omega) \omega_{i}^{\prime}-\omega^{2}\right)-m_{s} \frac{w}{t_{s}} \frac{j \omega Z_{L} C_{s}}{1+j \omega Z_{L} C_{s}} \frac{e_{31}^{2}}{\rho C_{s}} S_{1} S_{2}}
\end{aligned}
$$

where

$$
\begin{gathered}
\omega_{i}^{\prime 2}=\omega_{i}^{2}-m_{a} \frac{w}{t_{a}} \frac{e_{31} e_{31}}{\rho C_{a}} A_{1} A_{2}, \\
A_{1}=\int_{0}^{l} A_{a}^{\prime}(x) \phi_{i}(x) \mathrm{d} x, \\
A_{2}=\int_{0}^{l} A_{a}(x) \phi_{i}^{\prime}(x) \mathrm{d} x, \\
S_{1}=\int_{0}^{l} S_{a}^{\prime}(x) \phi_{i}(x) \mathrm{d} x, \\
S_{2}=\int_{0}^{l} S_{a}(x) \phi_{i}^{\prime}(x) \mathrm{d} x,
\end{gathered}
$$

$\omega_{i}$ is resonance frequency, $\omega^{\prime}$ is the anti-resonance frequency, $C_{a}$ is the static capacitance of the actuator, and $C_{s}$ is the static capacitance of the sensor. The products of $A_{1} A_{2}$ and $S_{1} S_{2}$ are negative in sign. For the case where no output electrode is present, i.e. a two-node component, $S_{1}$ and $S_{2}$ will be zero and the input impedance will only be the function of the actuator property. The term 'two-node component' means that the device has only one input node with a ground node, which is similar to the configuration used for quartz oscillators [11]. That is, for a two-node piezoelectric plate, its equivalent electrical impedance for bulk resonance will only be for the shape and location of the effective surface electrode. In other words, the input impedance is a function of the spatial domain and not merely a function of the material property and the structure resonance phenomenon. As for piezoelectric transformers, which are really a threenode component, its input impedance is determined from both the parameters of the actuator and sensor. The mentioned three-node component means that the device has one input and one output node compared to a common ground node. Therefore the input impedance of the piezoelectric transformer is determined by (1) static capacitance $C_{a}$, (2) the shapes of the electrodes $A_{a}(x)$ and $S_{s}(x)$, (3) output static capacitance $C_{s}$ and (4) loading impedance of the sensor. It should be mentioned that the loading impedance and the output static capacitance combine to form a high-pass filter that influences the distribution of the resonant and anti-resonant frequencies of the sensor output. More specifically, the real part of the this high-pass filter will shift the resonant and anti-resonant frequencies, and its imaginary part will reduce the quality factor or equivalently increase the equivalent damping ratio of the piezoelectric transformer. On the other hand, the highpass filter is influenced by the spatial property of the sensor, i.e. the output of the piezoelectric transformer, which depends on the shape and location of the effective surface electrode of the sensor. This understanding basically indicates that the input impedance can be tailored by choosing the shapes and location of the effective surface electrode, which leads to the potential of applying the modal sensor and actuator concept for the optimization of piezoelectric transformers. For the case where the output is open, i.e. the loading impedance $Z_{L}$ is approximated to infinity in equation (17), the input impedance can be rewritten as

$$
Z_{e}=\sum_{i=1}^{\infty} \frac{-1}{j \omega c_{a}} \frac{\left(\bar{\omega}_{i}^{2}+2 \zeta(j \omega) \bar{\omega}_{i}-\omega^{2}\right)}{\left(\bar{\omega}_{i}^{\prime 2}+2 \zeta(j \omega) \bar{\omega}_{i}^{\prime}-\omega^{2}\right)},
$$

where

$$
\begin{gathered}
\bar{\omega}_{i}^{2}=\omega_{i}^{2}-m_{s} \frac{w}{t_{s}} \frac{e_{31}^{2}}{\rho C_{s}} S_{1} S_{2}, \\
\bar{\omega}_{i}^{\prime 2}=\omega_{i}^{2}-m_{a} \frac{w}{t_{a}} \frac{e_{31}^{2}}{\rho C_{a}} A_{1} A-m_{s} \frac{w}{t_{s}} \frac{e_{31}^{2}}{\rho C_{s}} S_{1} S_{2} .
\end{gathered}
$$

It is clear from equation (20) that the resonant and antiresonant frequencies of the input impedance transfer function are increased by the open-circuit condition of the piezoelectric output. On the other hand, the output will not influence the input impedance at the short-circuit condition, i.e. the loading impedance $Z_{L}$ set to zero in equation (17). In this case, the input impedance is determined by the actuator itself. That is, the piezoelectric transformer will stiffen when the output is open, and will soften when the output is short [11]. More specifically, the piezoelectric transformer is really a two-node device when the output is short, and becomes a three-node device when the output has a piezoelectric effect [11]. In other words, the effect of the electric loading impedance is similar to a kind of clamped mechanical boundary condition, i.e. the rigidity of the clamping will change according to the value of the loading impedance. More specifically, the loading impedance will alter the motion of the piezoelectric transformer through the boundary effect. With this understanding, it should be noted that the 
mechanical quality factor of the piezoelectric transformer must be measured when the output is kept short.

The transfer function of this SISO piezoelectric transformer can be derived from equation $(16 b)$ as

$$
\begin{aligned}
\frac{V_{3 s}^{\text {out }}}{V_{3 a}^{\text {in }}} & =\sum_{i=1}^{\infty} \frac{j \omega Z_{L} C_{s}}{1+j \omega Z_{L} C_{s}} \\
& \times \frac{m_{a} \frac{w}{t_{s}} \frac{e_{31}^{2}}{\rho C_{s}} A_{1} S_{2}}{\left(\omega_{i}^{2}+2 \zeta(j \omega) \omega_{i}-\omega^{2}\right)-m_{s} \frac{w}{t_{s}} \frac{j \omega Z_{L} C_{s}}{1+j \omega Z_{L} C_{s}} \frac{e_{31}^{2}}{\rho C_{s}} S_{1} S_{2}} .
\end{aligned}
$$

It is clear from equation (22) that the influence of the output electrode to the resonance frequency and the damping ratio of the transfer function are identical to that of the input impedance. It should be indicated that the high-pass filter formed by the output static capacitance and loading impedance has two contributions to the transfer function in equation (22). The first one offers a high-pass filter to the overall transfer function of the SISO piezoelectric transformer. The second high-pass filter influences the quality factor and resonance frequency of the piezoelectric transformer, which is identical to the one in the input impedance. The resonance frequency will increase to the value predicted by equation (21a) with the output kept open, and becomes $\omega_{i}$ with the output in a short-circuit condition. On the other hand, the high-pass filter formed by the combination of the output capacitance $C_{S}$ and the loading impedance $Z_{L}$ in equation (22) will alter the phase distribution of the transfer function as well. Furthermore, the step-up ratio of the piezoelectric transformer at resonance frequency in equation (22) is a function of $A_{1} S_{2}$, which means the transfer function can be tailored by choosing the shapes and locations of the actuator (input section) and the sensor (output section) as defined by the effective surface electrodes.

In summary, piezoelectric transformers should be considered as an electrically as well as mechanically fully coupled system. Its governing equation is a PDE, i.e. it varies with parameters relating to both time and spatial domain. Furthermore, the input impedance and the transfer function depend on the mechanical and the electrical boundary conditions. That is, the motion of the piezoelectric transformer is determined by these boundary conditions. Since the piezoelectric transformer is a mechanical device, its equivalent electrical property must be a spatial function. Thus the previous original analysis methodology based on an equivalent circuit determined by an ODE $[1,7]$ cannot fully analyze the physical properties of transformers accurately. On the basis of these conclusions, an optimization of a SISO piezoelectric transformer will be detailed next.

\section{Optimization of piezoelectric transformers}

Since a piezoelectric transformer is a power transfer device, i.e. a three-node device, the requirements of its interface circuit are different from those used in typical two-node devices such as sensors and actuators. Since the resonance frequency and step-up ratio of the piezoelectric device depends strongly on loading conditions, the resonance frequency of the piezoelectric transformer sweeps from high to low frequencies when the load impedance changes from high to low. For the case where CCFL is used as the load, and which has an opencircuit condition before the lamp is turned on and with a load impedance in the range of hundreds of kilo-ohms after the lamp is on, the step-up ratio of the corresponding piezoelectric transformer will drop from around 2500 to 5, i.e. reduced by one to two orders. The phenomenon where the step-up ratio of a piezoelectric transformer changes with the loading condition makes it the best CCFL driving device and is one of the fundamental reasons that many organizations are rushing to make piezoelectric transformers commercial products [15]. Many previous studies have concentrated on the maximum amount of power that can be injected and then outputted, i.e. converted for a specific-sized piezoelectric transformer. In the following two sections, the case where that maximum power optimization cannot be determined by the size alone will be detailed. It can be seen that optimizing the piezoelectric transformer for maximum power transfer can be achieved by matching the electrical impedance of the input and output impedance, and by matching the modal strain of the structure resonance by the shape of the electrodes.

\subsection{Maximum power transfer by impedance match}

The output impedance of a piezoelectric transformer can be solely determined by the static capacitance of the sensor, i.e. the output section. Considering the case where output impedance $\left|1 / j \omega C_{s}\right|$ equals the loading impedance $Z_{L}$ at resonance frequency, i.e. the optimization of the maximum output power transfer of the piezoelectric transformer is reached, then the input impedance and system transfer function of the piezoelectric transformer can be derived from equations (17) and (22) as

$$
\begin{gathered}
Z_{e}=\sum_{i=1}^{\infty} \frac{-1}{j \omega c_{a}} \frac{\left(\overline{\bar{\omega}}_{i}^{2}+2 \zeta^{\prime}(j \omega) \overline{\bar{\omega}}_{i}-\omega^{2}\right)}{\left(\overline{\bar{\omega}}_{i}^{\prime 2}+2 \zeta^{\prime}(j \omega) \overline{\bar{\omega}}_{i}^{\prime}-\omega^{2}\right)}, \\
\frac{V_{3 s}^{\text {out }}}{V_{3 a}^{\text {in }}}=\sum_{i=1}^{\infty} \frac{j \omega Z_{L} C_{s}}{1+j \omega Z_{L} C_{s}} \frac{m_{a} \frac{w}{t_{s}} \frac{e_{31}^{2}}{\rho C_{s}} A_{1} S_{2}}{\left(\overline{\bar{\omega}}_{i}^{2}+2 \zeta^{\prime}(j \omega) \overline{\bar{\omega}}_{i}-\omega^{2}\right)},
\end{gathered}
$$

where

$$
\begin{gathered}
\overline{\bar{\omega}}_{i}^{2}=\omega_{i}^{2}-m_{s} \frac{1}{2} \frac{w}{t_{s}} \frac{e_{31}^{2}}{\rho C_{s}} S_{1} S_{2}, \\
\overline{\bar{\omega}}_{i}^{\prime 2}=\omega_{i}^{2}-m_{a} \frac{w}{t_{a}} \frac{e_{31}^{2}}{\rho C_{a}} A_{1} A-m_{s} \frac{1}{2} \frac{w}{t_{s}} \frac{e_{31}^{2}}{\rho C_{s}} S_{1} S_{2}, \\
2 \zeta^{\prime}(j \omega) \overline{\bar{\omega}}_{i}=2 \zeta(j \omega) \omega_{i}-m_{s} \frac{j}{2} \frac{w}{t_{s}} \frac{e_{31}^{2}}{\rho C_{s}} S_{1} S_{2} .
\end{gathered}
$$

It is clear from equation (24) that the high-pass filter at maximum power transfer condition raises both the resonance frequency and the damping ratio. This shows that the optimal operating condition of the piezoelectric transformer depends on both the mechanical and electrical characteristics of the piezoelectric transformer. Its transfer function at this condition is determined by the additional frequency response of the highpass filter from the output electrode, and the reduction of the quality factor and resonance frequency is a function of the shape and location of the sensor. The equivalent circuit used to model the motion of the piezoelectric transformer cannot express this condition completely as the spatial information is eliminated. Mechanically, a piezoelectric transformer is an electrical and mechanical fully coupled system and its 
mechanical motion is determined by both the mechanical and electrical boundary conditions. On the other hand, the equivalent electrical property is also determined by both the mechanical and electrical boundary conditions. Thus the variation of the loading conditions will influence its input impedance and transfer function. The reduction of both the quality factor and the resonance frequencies means that the power sinking of the loading impedance forms a damper to the structure. It must be mentioned that at a maximum power transfer of the operating resonance frequency, the high-pass filter will introduce a $45^{\circ}$ phase lead to the transfer function as the output impedance $\left|1 / j \omega C_{S}\right|$ equals $Z_{L}$ in equation (23). Furthermore, the step-up ratio will be filtered by this high-pass filter as its loading impedance is much lower than the output impedance of the piezoelectric transformer.

Since the mechanical quality factor of the piezoelectric transformer is up to 2000, the second term in equation (24c) will be much higher than the first term. The transfer function of the piezoelectric transformer operating at the resonance frequency can then be approximated as

$$
\left|\frac{V_{3 s}^{\text {out }}}{V_{3 a}^{\text {in }}}\right|=\left|\sqrt{2} \frac{m_{a} A_{1}}{m_{s} S_{1}}\right| .
$$

Note that the ratio $m_{a} / m_{s}$ and the shapes of the actuator and sensor determine the step-up ratio of the piezoelectric transformer. As the shapes of the sensor and actuator are selected according to modal sensor and actuator concept, the step-up ratio can only be determined by the ratio $m_{a} / m_{s}$. The required step-up ratio of a piezoelectric transformer can thus be easily implemented by using a multi-layer manufacturing process. For a single-layer piezoelectric transformer where $m_{a} / m_{s}$ equals one, its step-up ratio will be fixed at $\left|\sqrt{2} A_{1} / S_{1}\right|$. Optimizing the impedance match of the piezoelectric transformer for maximum power transfer is achieved by matching the output impedance to the loading impedance, which can be done by tuning the output static capacitance with a selected number of layers for the sensor portion, with the number of layers for the actuator as being determined by the required step-up ratio as per the above derivations. It should be noted that the capacitance of the actuator section is typically set to match the output impedance of the driving electronics, which is typically at $50 \Omega$ for standard signal electronics and is set at close to zero for power electronic applications. Thus the optimal electrical condition of a piezoelectric transformer is at its maximum power transfer. In the next section, the concept of matching the modal strain will be introduced to optimize the mechanical coupling of piezoelectric transformers.

\subsection{Optimizing sensors and actuators by matching modal strain}

The concept of modal sensors and actuators was first announced in the late 1980s [8] and its fundamental design concept was to match the modal strain of a specific mode by shaping the effective surface electrode to deal with the spillover in the flexible structural control. Since the shaped electrode in equation (15) offers a weighting function to the eigenfunction $\phi_{i}(x)$, the orthogonality condition of eigenfunctions can
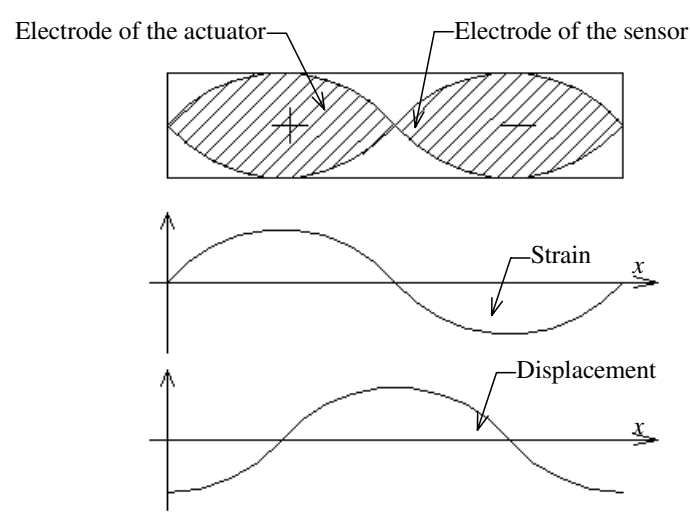

Figure 4. Schematic of a two-mode piezoelectric transformer, which has the effective surface electrode of an actuator and the sensor, matches the strain field of mode two.

be adopted to design modal sensors and actuators. More specifically, since

$$
\int_{0}^{l} \phi_{i}(x) \phi_{j}(x) \mathrm{d} x=1, \quad \text { for } i=j,
$$

and equals zero if $i \neq j$, choosing $A_{a}^{\prime}(x)=\phi_{i}(x)$ and $S_{s}(x)=$ $\phi_{i}^{\prime}(x)$ for $A_{1}$ and $S_{2}$ in equation (22) leads to the conclusion that only the $i$ th resonance mode will be actuated and sensed by the piezoelectric transformer. With this condition, the piezoelectric transformer will act like a single-mode resonance tank with only one resonance frequency present. Once this condition is achieved, the piezoelectric transformer can be expressed fully by using an ODE. That is, once the modal sensor and actuator concept is adopted, a spatially dependent equivalent circuit can be adopted to study the electric behavior of the piezoelectric transformer, which is similar to the traditional approach adopted. It can be understood that once the equivalent circuit concept is correctly reintroduced back into the design of the piezoelectric transformer, the interface circuits needed to operate the piezoelectric transformer at its fullest operating regime can be realized.

Figure 4 details the schematics of a piezoelectric transformer which has the effective surface electrode of the actuator portion matching the left half period of the mode two strain field, and sensor portion matching that of the right half section. Since the piezoelectric transformer is operated on the chosen resonance frequency, its strain distribution across the whole piezoelectric transformer will be the modal strain field of the operated mode. More specifically, shaping the actuator electrode to match the modal strain distribution will lead to the condition where the flow of the electrical energy will only be spread into the specific mode chosen. On the other hand, a shaped modal sensor will only sink the energy generated from this mode. An optimized coupling of an energy transfer between the mechanical motion and interface circuits can then be achieved. In summary, an optimized piezoelectric transformer with a maximum power transfer is comprised of two parts: first, it matches its loading impedance with a designed output impedance, and secondly, the concept of the modal sensor and actuator are adopted to match the desired modal strain distribution. Experimental data obtained which verify the validity and effectiveness of the above-mentioned concept will be presented. 


\section{Experimental set-up}

A $44 \mathrm{~mm}$ long, $6.5 \mathrm{~mm}$ wide, and $22 \mathrm{~mm}$ thick single-layer piezoelectric transformer (figure 4) was used to verify the theoretical predictions: i.e. $m_{a}$ and $m_{s}$ equal one, since a single-layered sensor and actuator were used. This transformer was designed to operate in the second mode, and was made with PZT material. Since the quality factor of the piezoelectric transformer is about 1800 and the PZT adopted is closer to that of the PZT- 8 series, the material constant of the PZT- 8 series was used to compute the theoretical value. That is, the piezoelectric stress constant used was $e_{31}=-4 \mathrm{cl} \mathrm{m}^{-2}$ and density $\rho=7600 \mathrm{~kg} \mathrm{~m}^{-3}$. The structure resonance and anti-resonance frequency $f_{i}$ and $f_{i}^{\prime}$ were at $77.936 \mathrm{kHz}$ and $79.905 \mathrm{kHz}$ from the resonance frequency of the input impedance measured using an Agilent 4294A [16] impedance analyzer when the output was connected short. The input and output capacitances were measured by using an Agilent 4294A impedance analyzer and were found to be around $520 \mathrm{pF}$, which translates to a theoretical optimized loading impedance of around $4.1 \mathrm{k} \Omega$. Resistors were then used to serve as the desired loading impedance. With the values mentioned above, the theoretical input impedance and transfer function as predicted by equations (17) and (22) become

$$
\begin{aligned}
Z_{e} & =\sum_{i=1}^{\infty} \frac{-1}{j 2 \pi f \times 520 \mathrm{pF}} \\
& \times \frac{\left(f_{i}^{2}+j\left(f f_{i} / 1800\right)-f^{2}\right)+H(f) 343.512164 \times 10^{6}}{\left(f_{i}^{\prime 2}+j\left(f f_{i}^{\prime} / 1800\right)-f^{2}\right)+H(f) 343.512164 \times 10^{6}}
\end{aligned}
$$

$$
\begin{aligned}
& \frac{V_{3 s}^{\text {out }}}{V_{3 a}^{\text {in }}}=\sum_{i=1}^{\infty} H(f) \\
& \quad \times \frac{343.512164 \times 10^{6}}{\left(f_{i}^{2}+j\left(f f_{i} / 1800\right)-f^{2}\right)+H(f) 343.512164 \times 10^{6}},
\end{aligned}
$$

where

$$
H(f)=\frac{j 2 \pi f(520 \mathrm{pF}) Z_{L}}{1+j 2 \pi f(520 \mathrm{pF}) Z_{L}},
$$

is the high-pass filter formed by the loading impedance and output static capacitance. Figure 5 shows the experimental results of the input impedance obtained by the Agilent 4294A impedance analyzer: the thin dark curves represent the results for the condition where the output is open and at a $56 \Omega$ loading condition, and the grey curves represent the corresponding theoretical values. The impedance was measured at resonance $\left(F_{r}\right)$ and anti-resonance $\left(F_{a}\right)$ frequencies with loading impedance varied from open to short, and was performed by placing different precision resistors of different values at the output sections of the piezoelectric transformer as shown in figure 5 (thick dark curve). It is obvious from figure 5 that the equivalent damping ratio will increase significantly as the loading impedance approaches the output impedance, and recover as the loading impedance deviates further with the output impedance. On the other hand, the phases of the resonance and anti-resonance frequency will drop about $45^{\circ}$ at $4.12 \mathrm{k} \Omega$. This phenomenon can be easily understood by considering the physically meaning. More specifically, the difference of the resonance impedances among
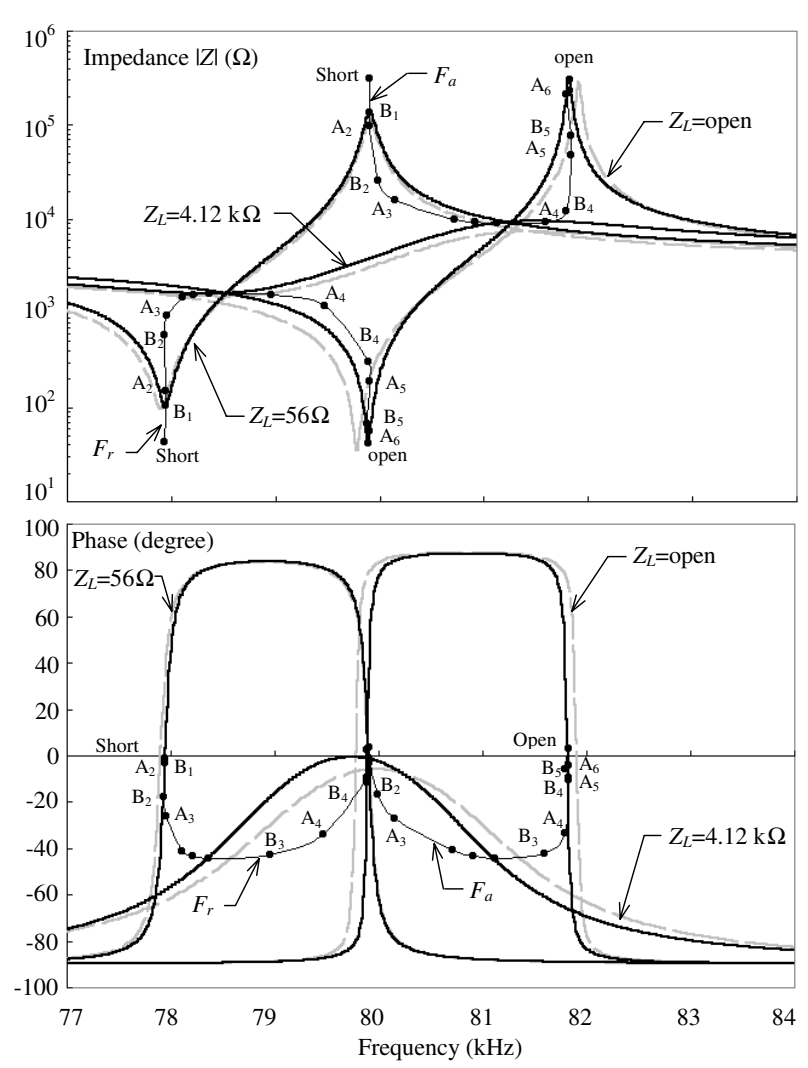

Figure 5. Input impedance of the piezoelectric transfer: (a) experimental results (dark curve), (b) theoretical values (grey curve) and (c) impedance measured at the resonance $\left(F_{r}\right)$ and anti-resonance $\left(F_{a}\right)$ frequencies when the loading impedance $\left(A_{i}=10^{i}, B_{i}=5.6 \times 10^{i}\right)$ varies from an open- to short-circuit condition at the output electrode section (thick dark curve).

short, open and optimal loading conditions can all be attributed to be the influence of the loading impedance which sinks out power of the piezoelectric transformer driving circuit. In other words, the higher the resonance impedance rises due to the loading impedance, the more the power is converted and transferred by the piezoelectric transformer. It should be noted that the input impedance contributed by the piezoelectric transformer is around $50 \Omega$, which means that it consumes little power as a transformer. This is the fundamental reason why the efficiency of a piezoelectric transformer is much better than that of electromagnetic transformers. From the experimental results shown in figure 5, it should be clear why the optimal loading impedance of a piezoelectric transformer equals its output static impedance.

Figure 6 shows the experimental results of a transfer function using an Agilent 4395A [17] network analyzer: the thin dark curves are the results obtained when the output is $100 \mathrm{M} \Omega$ and $56 \Omega$ and the grey curves are the corresponding theoretical values. The variations of the gain and phase for the resonance frequency with loading impedance varying from $100 \mathrm{M} \Omega$ and $56 \Omega$ is shown in figure 6 as a thick dark curve. It is obvious from figure 6 that the equivalent damping ratio will also increase as the loading impedance approaches the output impedance and decrease as they become further apart. On the other hand, the phase of the resonance and anti-resonance frequency will rise about $45^{\circ}$ at $4.12 \mathrm{k} \Omega$, and shows an almost $90^{\circ}$ phase lead as output approaches close 

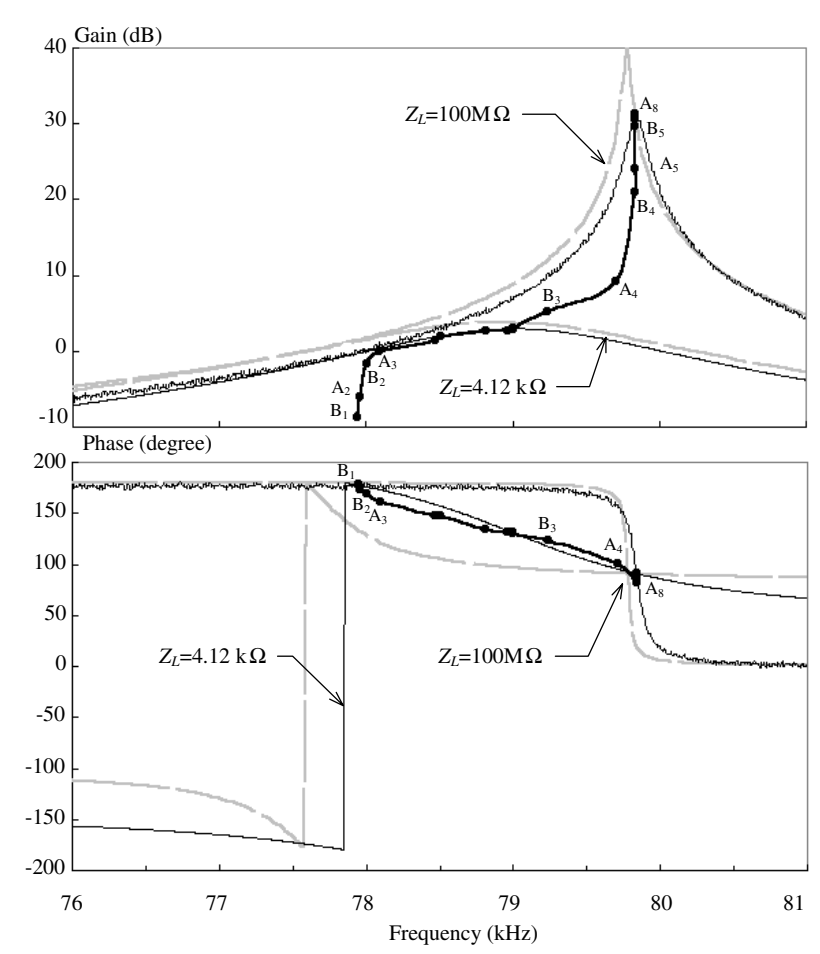

Figure 6. Transfer function of the piezoelectric transfer: (a) experimental results (dark curve), (b) theoretical values (grey curve), and (c) gain and phase measured at the resonance frequency with loading impedance $\left(A_{i}=10^{i}, B_{i}=5.6 \times 10^{i}\right)$ varying from $100 \mathrm{M} \Omega$ to $56 \Omega$ (thick dark curve).

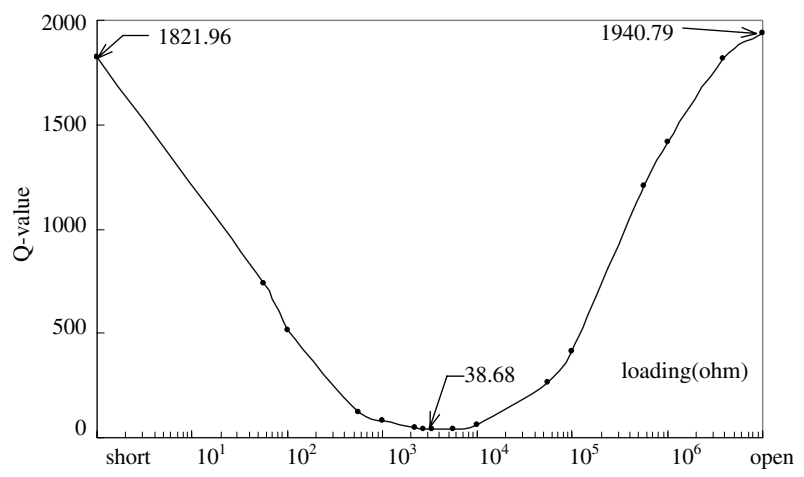

Figure 7. Fitted quality factor curve of the input impedance with varying loading impedance.

to the short-circuit condition, which can be attributed to the influence of the high-pass filter $H(f)$. The step-up ratio of this piezoelectric transformer is $2.839744797 \mathrm{~dB}$, which fits the theoretical value $3 \mathrm{~dB}$, or $2^{1 / 2}$ in magnitude, from the theoretical predictions of equation (25). This phenomenon matches the well-known effect where the step-up ratio and resonance frequency of a piezoelectric transformer will drop when the loading impedance is reduced, which enables the piezoelectric transformer to serve as the best driving circuit for plasma-based illumination devices such as CCFL and CFL tubes. Figure 7 is the quality factor of the input impedance curve fitted with the data obtained by using the Agilent 4294A [16] with various loading impedances ranging from 1940.79 and 1821.96 as the output was set at an open- and short-circuit condition. The mechanical quality factor of a

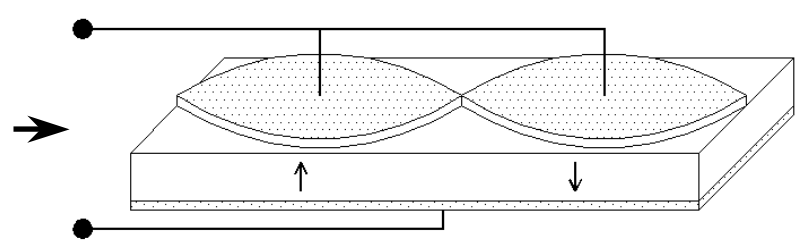

Figure 8. Piezoelectric plate designed by adopting modal strain-based surface electrodes.

piezoelectric transformer should be measured at the condition when the output is short as the electrical dependency of the piezoelectric material then disappears. The experimentally measured quality factor for piezoelectric transformers is up to 1821.96 . On the other hand, the quality factor drops to 38.68 for the case of matching output-loading impedance. It should be mentioned that both the input impedance and transfer function of the piezoelectric transformer has a $45^{\circ}$ phase shift at an optimal loading impedance. This is significantly different from traditional thoughts regarding the electrical property of an equivalent RLC circuit and the mechanical resonance phenomenon. The traditional equivalent circuit, which is based on a resonance tank model alone, cannot be used to describe the motion of piezoelectric transformers fully at an optimal loading condition. The influence of the high-pass filter in equation (28) is weighted by the spatial dependence of the sensor electrode, i.e. the equivalent circuit represents the spatially averaged effect. It cannot thoroughly express the spatial dependency of a piezoelectric transformer at optimal condition. More specifically, the resonance property of a mechanical structure will drop about $180^{\circ}$ for each mode, which is different from the $45^{\circ}$ phase lag. However, the transfer function at this condition, i.e. the quality factor and resonance frequency, is determined by the mechanical and electrical properties simultaneously. This discrepancy can be traced to the influence of the spatially weighted high-pass filter formed by the output impedance and the load, which imposes its effect on the transfer function of the piezoelectric transformer at the operating condition. In other words, a piezoelectric transformer must be considered as an electrical as well as a mechanical fully coupled structure system. Mechanically, its motion is determined by both the electrical and mechanical boundary conditions. Electrically, there is a high-pass filter formed at the output of the piezoelectric transformer, which must be carefully embedded into the equivalent circuit of the piezoelectric transformer. In other words, both the input impedance and frequency response of the piezoelectric transformer represent the coupling effect, and the phase shift demonstrates how much these two physical properties couple with each other.

To verify the concept of using modal actuators and sensors to match specific information of a structural mode, an impedance of a piezoelectric plate like the one shown in figure 8 was used. Figure 9 shows the experimental results of the piezoelectric plate (dark curve) when compared to the actuator used in figure 4 (grey curve). It is obvious from figure 9 that the input impedance of a full modal electrode will filter out other structure mode information, and its electrical property is equivalent to an RLC-C resonance tank. That is, once the modal sensors and actuators are adopted, a piezoelectric transformer can be modeled by using 

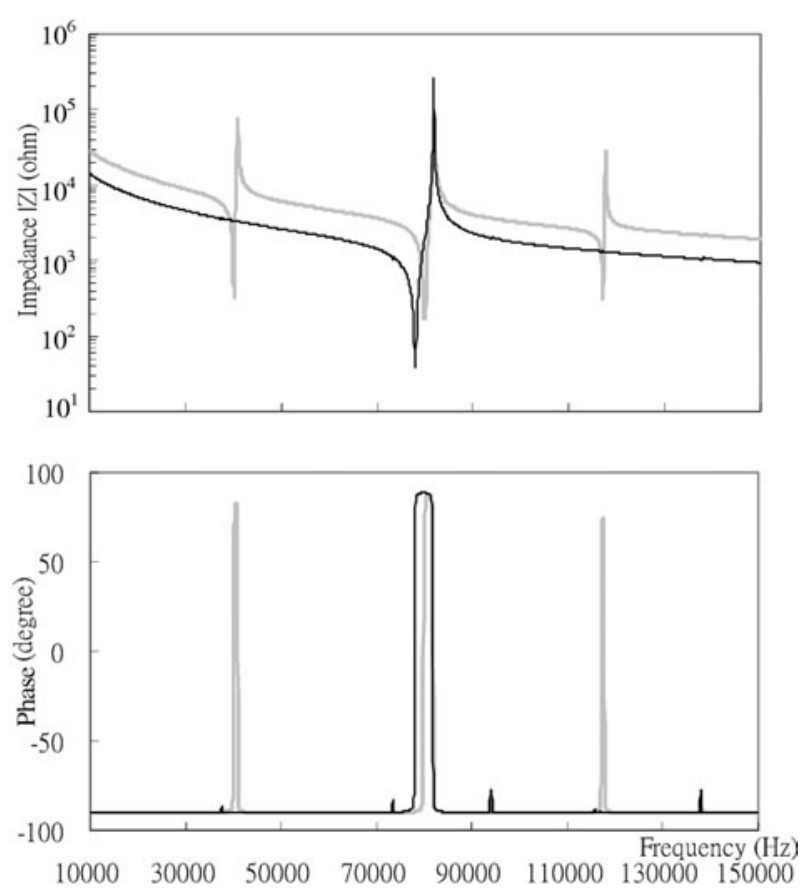

Figure 9. Impedance of a piezoelectric plate with full modal electrode (dark curve) and half modal electrode (grey curve).

an ODE, i.e. the traditional equivalent circuit approach can then be reintroduced to design the interfacing circuit needed.

\section{Conclusions}

Both theoretical and experimental results were shown to verify the optimization requirements of a piezoelectric transformer. A generalized governing equation of a one-dimensional multilayer piezoelectric transformer with multi-input and multioutput was derived to study the motions of the piezoelectric transformer. A SISO piezoelectric transformer was used to verify the concept of optimizing a piezoelectric transformer by introducing an impedance match as well as the concept of modal actuators and sensors. Steps to optimize the power transfer of a piezoelectric transformers are identified as

(1) shape the electrodes of the sensors and actuators to match the modal strain of the operating resonance mode,

(2) match the loading impedance that sinks the power of piezoelectric transformer with $m_{s}$ layers, and

(3) choose $m_{a}$ layers of an actuator to match the desired stepup ratio from the $m_{a} / m_{s}$ relationships.

Note that the piezoelectric transformer mentioned in this paper can be used to perform a step-up or step-down function for an $\mathrm{AC}$ power source in order to achieve the voltage and current for different loading conditions when an optimal power transfer condition is to be approached. The coupling effects between the electrical and mechanical physical phenomena were well modeled using the appropriate PDEs. Taking this approach, both the influence of the mechanical motion in the spatial domain and electrical loading in the time domain relative to the operation of piezoelectric transformer can be presented by using the corresponding field equation, which must be spatially and time dependent. As the field equation is a PDE in nature, it also indicates that an equivalent RLC circuit, which is an ODE, cannot represent these effects fully.

In summary, the theoretical derivations and experimental data obtained in this paper demonstrate clearly that a piezoelectric transformer must be considered as an electrical as well as a mechanical fully coupled system. The loading impedance can alter the motion of the piezoelectric transformer and can leave its mark on the input impedance and the frequency response. It was shown that the impedance of the input resonance frequency will rise to the maximum value at the optimal loading impedance, and this additional impedance represents the consumption induced by the extra loading of the driving circuit. On the other hand, a $45^{\circ}$ phase shift was found to be present when the optimal condition is achieved, which can be approached by introducing the proper input impedance and frequency response. Finally, the concept of modal sensors and actuators was introduced to optimize the power transfer between the electrical and mechanical energies. It was also verified experimentally that a full modal electrode offers a spatial weighting function that can filter out the contribution from other structural resonance modes. Furthermore, the piezoelectric transformer can be modeled as a single RLC-C resonance tank in this condition. In summary, optimization of a piezoelectric transformer can be realized both mechanically and electrically.

\section{Acknowledgments}

The authors would like to acknowledge the continuous and generous financial support of this research from the National Science Council of Taiwan, ROC, and from AHEAD Optoelectronics, Inc. through Grants NSC 85-2622-E-002017R, NSC 86-2622-E-002-023R, NSC 88-2218-E-002-005, and NSC 88-2622-E-002-001. The financial and material support of AdvanceWave Technology, Inc. is also greatly appreciated as well. Thanks are due also to Eleceram Technology Co. Ltd for providing us with the piezoelectric transformers used in this research work.

\section{References}

[1] Rosen C A 1956 Ceramic transformers and filters Proc. Electric Comp. Symp. pp 205-11

[2] Rosen C A 1958 Piezoelectric transformers US Patent Specification 2830274

[3] Kazimierczuk M K and Czarkowsi D 1995 Resonant Power Converters (New York: Wiley)

[4] Kawai J and Tagami B 1994 Piezoelectric transformer and method for driving the same US Patent Specification 5365141

[5] Kanayama K and Maruko N 1995 Piezoelectric transformer US Patent Specification 5701049

[6] Tooru A 1996 Piezoelectric transformer and power converting apparatus employing the same US Patent Specification 5751092

[7] Katz H W 1959 Solid State Magnetic and Dielectric Devices (New York: Wiley) pp 94-126

[8] Lee C K and Moon F C 1990 Modal sensors/actuators ASME J. Appl. Mech. 57 434-41

[9] Cady W G 1964 Piezoelectricity vol 1 (New York: McGraw-Hill) pp 1-8

[10] ANSI/IEEE Standard 1761987 Piezoelectricity (Piscataway, NJ: IEEE)

[11] Tiersten H F 1969 Linear Piezoelectric Plate Vibrations (New York: Plenum) 
[12] Horowitz P and Hill W 1998 The Art of Electronics (New York: Cambridge University Press)

[13] Royer D and Dieulesaint E 1996 Elastic Waves in Solids vol 1 (New York: Springer)

[14] Meirovitch L 1986 Element of Vibration (Singapore: McGraw-Hill)

[15] Li C Y 1997 Design and analysis of piezoelectric transformer converters PhD Thesis Virginia Polytechnic Institute
[16] Agilent 4294A Precision Impedance Analyzer Operation Manual Agilent Technologies Japan, Ltd, Component Test PGU-Kobe 1-3-2, Murotani, Nishi-ku, Kobe-shi, Hyogo, 651-2241 Japan, January 2001

[17] Agilent 4395A Network/Spectrum/Impedance Analyzer Operation Manual Agilent Technologies Japan, Ltd Component Test PGU-Kobe 1-3-2, Murotani, Nishi-ku, Kobe-shi, Hyogo, 651-2241 Japan, March 2000 